\title{
Angle Sensors Based on Oblique Giant Magneto Impedance Devices
}

\author{
Dohun Kim, Jiwon Na, and Won Young Jeung* \\ Functional Materials Research Center, Korea Institute of Science and Technology, Hawolgok Dong, Seongbuk Gu, Seoul 136-791, Korea
}

(Received 7 November 2008, Received in final form 3 February 2009, Accepted 4 February 2009)

\begin{abstract}
The measurement of external magnetic field orientation using Giant Magneto Impedance (GMI) sensors has been performed. A soft magnetic alloy of $\mathrm{Co}_{30} \mathrm{Fe}_{34} \mathrm{Ni}_{36}$ was electroplated on a $\mathrm{Si}$ wafer with a $\mathrm{CoFeNi}$ seed layer. V-shaped microwire patterns were formed using a conventional photolithography process. An external magnetic field was generated by a rectangular AINiCo permanent magnet. The reference direction was defined as the external magnetic field direction oriented in the middle of 2 GMI devices. As the orientation of the magnetic field deviated from the reference direction, variation in the field component along each device introduced voltage changes. It was found that, by taking the voltage difference between the left and right arms of the $\mathrm{V}$ shaped device, the nonlinearity of each device could be significantly reduced. The fabricated angle sensor had a linear range of approximately $70^{\circ}$ and an overall sensitivity of approximately $10 \mathrm{mV}$.
\end{abstract}

Keywords : Giant Magneto Impedance (GMI) effect, uniaxial anisotropy, magnetic sensor, differential amplification, linear response

\section{Introduction}

Since the discovery of the Giant Magneto Impedance (GMI) effect, in which the impedance of a high-permeability element changes with an external dc field due to the skin effect at a high-frequency current, it has been an attractive research field for experimental and theoretical investigations given its high sensitivity, resolution, and low power consumption [1-3]. First discovered in zero magneto-restrictive amorphous wires, magneto impedance ratios from $>10 \%$ to $>100 \%$ have been demonstrated using amorphous ribbons, nanocrystalline wires, and multilayered structures [4-7]. However, since the GMI effect is closely related to the magnetic permeability of the soft magnetic material, the impedance response to an external magnetic field basically exhibits a non-linear and symmetric behavior that can be problematic in practical applications. In order to obtain a linear response, a number of approaches have been proposed. P. Ripka and L. Kraus applied an antiparallel bias field to 2 amorphous magneto impedance sensors and amplified the voltage difference [6]. In addition, K. Mohri et al. used a pulse signal with an integrated multivibrator circuit and feedback coil technique in order to obtain an asymmetrical MI effect

*Corresponding author: Tel: +82-2-958-5422

Fax:+82-2-958-6839, e-mail: wyjeung@kist.re.kr and linear response, respectively [7].

The application of linear response magnetic field sensors based on the GMI effect, as mentioned in Ref 7, is diverse. Apart from obvious application of GMI sensors to magnitude detection of a magnetic field, adopting a permanent magnet near the GMI sensor and reading signal variation enables the detection of various physical quantities, including position, acceleration, and orientation. In particular, for the angle sensor application, a common method is to detect the field component of 2 orthogonal directions, namely the $\mathrm{x}$ - and $\mathrm{y}$-axes, and compute the arc tangent of their ratio. This detection scheme can be beneficial when the full range of 360 degrees is required. However, the structural complexity and the generation of the bias field by microcoils require high power consumption.

In this work, it was demonstrated that, using 2 obliquely-laid GMI devices and an external permanent magnet, the generated magnetic field acts as a bias field so that the voltage difference between the 2 oblique GMI devices becomes linear to the orientation of the magnetic field when the field orientation lies between the 2 GMI devices. Although it has a limited range of linear response, it is expected that the simplicity of the device structure and no necessity of bias coils significantly reduce power consumption. 


\section{Experiments}

The device pattern for the V-shaped GMI devices were formed on a Si wafer by a photolithography method. The CoFeNi alloy was sputtered as a seed layer by a lift off process. In previous works [7-10], we have reported the effect of electroplating conditions on the soft magnetic properties of $\mathrm{CoFeNi}$ alloys. The optimal composition was found to be $\mathrm{Co}_{30} \mathrm{Fe}_{34} \mathrm{Ni}_{36}$ and the saturation magnetic-flux density, permeability, and coercivity field were approximately $1.9 \mathrm{~T}, 3.4 \times 10^{6}$, and $0.1 \mathrm{Oe}$, respectively. Using these conditions, a soft magnetic alloy with a thickness of $1.0 \mu \mathrm{m}$ was electroplated on a CoFeNi sputtered Si wafer. Microwire patterns had a width of $100 \mu \mathrm{m}$ and a length of $4000 \mu \mathrm{m}$.

The angle between the 2 devices determined the linear response range of the sensor. As it can be expected from the sensing principle (Fig. 2), orthogonal configurations lead to a maximum angle detection range of 90 degrees. However, it is well known that transverse anisotropy is prerequisite for a large GMI effect in the RF regime. From previous work [10], it was found that applying an external magnetic field orthogonal to the wire's direction during the electrodeposition process induces transverse anisotropy. Since, in the present experiment, the external magnetic field was applied orthogonal to the reference direction, defined as the direction of the centerline of the $\mathrm{V}$ shaped device, the resultant anisotropy of each device can be considered helical. Because excessive deviation from the orthogonal magnetic field application greatly reduces the GMI property, the angle between the 2 GMI devices was set to 50 degrees, thereby ensuring GMI characteristics at the expense of an angle detection range.

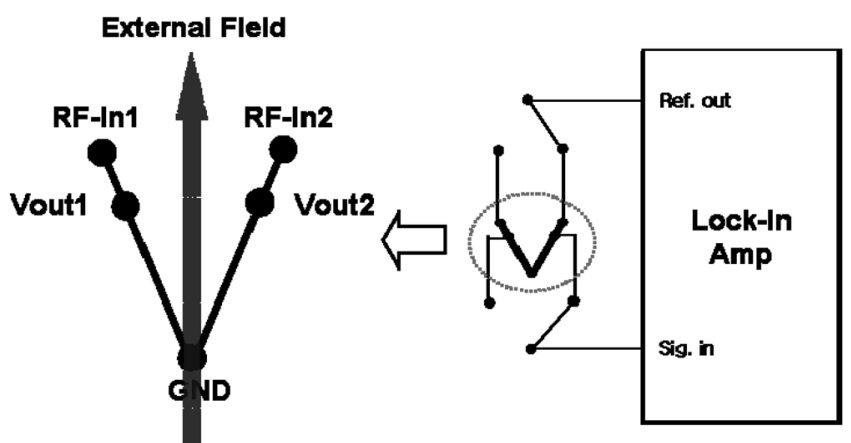

Fig. 1. Schematic diagram of the GMI sensor configuration and measurement system. The angle between the 2 GMI devices was set to $50^{\circ}$, thereby ensuring transverse magnetic anisotropy at the expense of the angle detection range. For the measurement of the 2 GMI devices, a multiplexer (SR3830) is used to switch voltage output of the GMI devices alternatively at a period of approximately $10 \mathrm{~ms}$ with the voltage output from each sensor subtracted.
Fig. 1 depicts schematics of the device configuration and measurement setup.

Electrical contacts for measurements were formed by sputtered gold electrodes. Gold wire with a diameter of $50 \mu \mathrm{m}$ was used for the interconnections between a device and the sample holder. After the fabrication of the devices, the soft magnetic properties of the devices were characterized by a Lakeshore 7400 vibrating sample magnetometer (VSM). From the hysteresis loop measurements on both the easy and hard axes, the coercivity of $\mathrm{Co}_{30} \mathrm{Fe}_{34} \mathrm{Ni}_{36}$ was found to be less than 0.2 Oe and the transverse magnetic anisotropy was confirmed with an anisotropy field of approximately $17 \mathrm{Oe}$.

Conventionally, for the determination of impedances at high frequencies, reflection coefficient measurements using network analyzers are commonly used. However, since it is the output voltage that is ultimately used for the sensor application, the sensor's root mean square (RMS) voltage signal was employed, both for magnetic impedance ratio evaluation and numerical linearization. A Stanford Research Systems SR844 High frequency Lock-in Amplifier was used as both a RF signal source and RMS voltage detector. As for the frequency range, because of the physical dimensions of the lab-built test fixture used $(5.0 \mathrm{~cm}$ from the front end of the SMA connectors to the sample), the valid measurement range was limited to approximately 50 $\mathrm{MHz}$ [10]. In this experiment, we used the reference square wave output of the lock in amplifier which had an amplitude of $1.0 \mathrm{Vpp}$ and a fundamental frequency of $20 \mathrm{MHz}$.

Firstly, GMI profiles with respect to the magnitude of the external magnetic field were measured for each device. From this measurement, the magnitude of the sample's anisotropy field was determined and an overall GMI ratio was calculated. After that, using a different measurement setup capable of rotating the permanent magnet around GMI devices, the GMI profile of each device with respect to the field orientation was measured. For the measurement of the 2 GMI devices, we used a multiplexer (SR3830) to alternatively switch the voltage output of the GMI devices at a period of about $10 \mathrm{~ms}$ and the voltage output from each sensor was subtracted. The time constant setting of the lock in amplifier and the rotational speed of the external magnetic field were set adequately so that the effect of the time difference of $10 \mathrm{~ms}$ could be neglected. As the frequency range in this work was well below the microwave regime, an intricate calibration using short, open, load, and through standards was not needed.

\section{Results and Discussion}

The origin of the GMI effect, when the exciting fre- 
quency exceeds well over a few $\mathrm{MHz}$, can be classified into 2 ranges depending on the frequency of the exciting ac signal. When the exciting frequency ranges from a few $\mathrm{MHz}$ to tens of $\mathrm{MHz}$, a severe eddy current inhibits the domain wall motion so that the magnetization rotation determines the magnetization process. Also, due to the skin effect, the applied electromagnetic field is concentrated at the surface of the material. This increased surface contribution largely modifies the impedance profile and the percentage variation increases to well over $10 \%$. Moreover, in the microwave regime above $\sim \mathrm{GHz}$, ferromagnetic resonance (FMR) contributes to the GMI profile. As our operating frequency was set to $20 \mathrm{MHz}$, it is expected that the GMI profile of the present devices stemmed from the skin effect and the transverse magnetic permeability response during magnetization rotation.

Fig. 2 shows the GMI profile of each device at frequencies of $20 \mathrm{MHz}, \mathbf{2 a}$, and the principle of obtaining the linear response with respect to magnetic field orientation, 2b. Like conventional GMI devices, our GMI sensor showed symmetric and nonlinear behavior with respect to the applied magnetic field. In this experiment, the GMI ratio was defined as follows:

$$
\operatorname{GMI}(\%)=\frac{V_{r m s}(H)-V_{r m s}(0)}{V_{r m s}(0)}
$$

At a frequency of $20 \mathrm{MHz}$, as the external magnetic field was swept from -85 to 85 Oe, the sensor output voltage varied about $10 \%$. In addition, as the operating frequency was above $10 \mathrm{MHz}$, the GMI profile showed clear double peak behavior, consistent with previous works $[2,5,6,12,13]$. Moreover, from the observation of the external field magnitude at the maximum device voltage, the effective magnetic anisotropic field for the transverse direction was determined to be near $17 \mathrm{Oe}$, a consistent value obtained with a VSM measurement. As shown in the figure, the measured GMI response shows almost zero hysteresis due to the ultra soft magnetic properties of the electroplated $\mathrm{Co}_{30} \mathrm{Fe}_{34} \mathrm{Ni}_{36}$ devices.

From the typical GMI profiles in the RF regime, the following method is taken to obtain linearized sensor output, Fig. 2b. At first, by adjusting the distance between the device and the permanent magnet, the magnitude of the magnetic field when the magnet is parallel with the right arm of the V-shaped GMI devices is set to approximately $16 \mathrm{Oe}$, slightly lower than the anisotropy field of the device (point A in Fig. 2b). At this point, the orientation of the magnetic field has an angle of $50^{\circ}$ with respect to the left arm of the device, so that the actual magnetic field along the left device's direction is only 16 Oe times $\cos 50^{\circ}$, near 10 Oe. Thus, the sensor output voltage from (a)
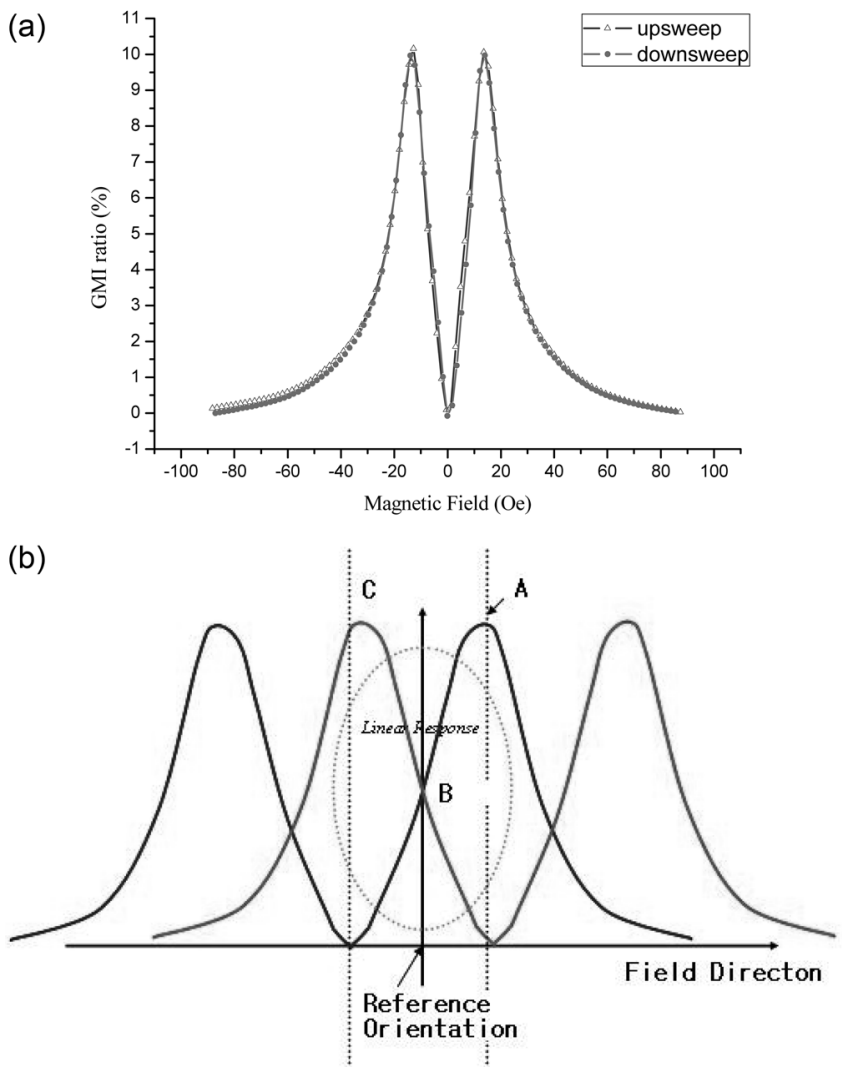

Fig. 2. (a) Original GMI profiles of the $\mathrm{Co}_{30} \mathrm{Fe}_{34} \mathrm{Ni}_{36}$ micropatterned devices at frequencies of $20 \mathrm{MHz}$; (b) principle of obtaining linear response.

the right arm is higher than that of the left. As the field orientation deviates from this direction, when the external field lies in the middle of the 2 arms, the voltage response of the 2 devices is the same. Moreover, as the magnetic field rotates towards the left arm, the voltage value reverses. Note that the 2 GMI devices are fabricated in the same electrodeposition bath so that the magnetic response and the degree of nonlinearity of the GMI profile with respect to the external field can be considered to be identical. Therefore, by taking the voltage difference from the 2 devices as the magnetic field rotates, the nonlinearities of each sensor can be significantly reduced and a linear response directly to the orientation of the external magnetic field is possible.

Fig. 3 shows the measured GMI profiles with respect to the magnetic field orientation (a) and the linear response of the voltage difference between the 2 GMI devices (b). As expected, although the output voltage of each device shows severe nonlinearity, the voltage difference exhibits a reasonable degree of linearity. In practice, the center of the external magnet cannot be perfectly aligned with the center (ground point) of the device. Moreover, the GMI characteristic, the maximum voltage value of the 2 devices, 

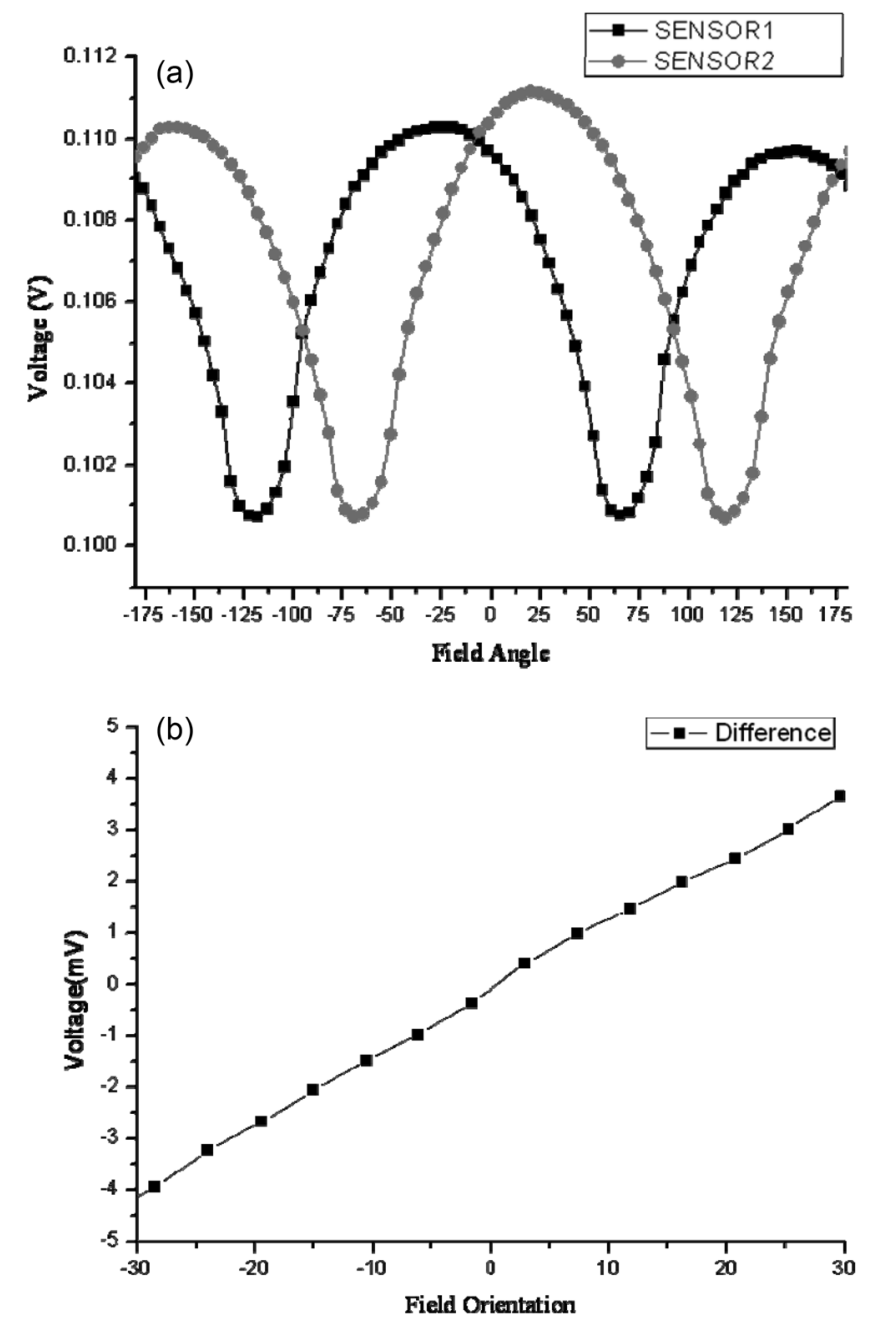

Fig. 3. Detailed GMI profiles with respect to magnetic field orientation: (a) voltage response of each device; (b) voltage difference, which shows a linear response from -30 to +30 magnetic field orientation.

shows a slight difference. The non-identical voltage maxima in Fig. 3a originate from these non-ideal limitations. However, as shown in Fig. 3b, the voltage difference of the 2 devices significantly reduces mutual error. With adequate tuning of each sensor, amplifying each sensor output with a different gain, it is expected that the remaining nonlinearity can be further reduced. The fabricated angle sensor shows a linear response from $-30^{\circ}$ to $30^{\circ}$ and a total sensitivity of close to $10 \mathrm{mV}$ without amplification. Using an instrumentation amplifier at the final stage and analog to digital conversion, acute detection of the magnetic field orientation is possible.

This method of obtaining the voltage response with respect to field orientation has several advantages over conventional methods in the sense that the need for the bias coil and feedback coil is eliminated. Moreover, the fact that the voltage output is directly proportional to field orientation significantly simplifies the later stage of the sensor system on the ground that the numerical computation of the arc tangent value is not needed. Obviously, this kind of sensor cannot be used for the determination of a full degree of orientation, such as an electronic compass. Instead, it can be applicable in the field as sensitive motion control around an equilibrium point. An angular control system for automotive applications is one example. In fact, it was found that the linear response range of the angle sensor of this kind significantly depends on such factors as the distance between the permanent magnet and the sensor or shape of the external magnet. With proper adjustment of these parameters, it is expected that the linear range can be enhanced over $0 \sim 90^{\circ}$, which covers the angular range of many automotive applications.

\section{Concluding Remarks}

In summary, linear response GMI devices were fabricated using 2 obliquely configured $\mathrm{Co}_{30} \mathrm{Fe}_{34} \mathrm{Ni}_{36}$ electroplated and patterned nanocrystalline microwires. Although each GMI device showed non-linear behavior with respect to external magnetic field orientation, subtracting voltage difference lead to a linear voltage response when the external magnetic field laid between 2 devices of the Vshaped angle sensor. It is expected that a linear response, directly to the external field direction, considerably simplify subsequent electronic circuitry.

\section{Acknowledgment}

This work was supported by a grant from the Fundamental R\&D Program for Core Technology of Materials, funded by the Ministry of Commerce, and R\&D Program for NT-IT Fusion Strategy of Advanced Technologies, Industry and Energy, Republic of Korea.

\section{References}

[1] L. V. Panina and K. Mohri, Appl. Phys. Lett. 65, 1189 (1994).

[2] M. Knobel, M. L. Sánchez, C. Gómez-Polo, P. Marín, M. Vázquez, and A. Hernando, J. Appl. Phys. 79, 1646 (1996).

[3] H. Chiriac, T. A. Óvári, and C. S. Marinescu, J. Appl. Phys. 83, 6584 (1998).

[4] Chen, C. et al., J. Phys. D 9, 1951 (1997).

[5] D. de Cos et al., 41, 3697 (2005).

[6] P. Ripka and L. Kraus, MA: Artech House, 350 (2001).

[7] K. Mohri, T. Uchiyamaa, L. P. Shena, C. M. Caia, and L. 
V. Panina, J. Magn. Magn. Matt. 249, 351 (2002).

[8] W. Y. Jeung, H. K. Kim, and J. O. Lee, J. of Kor. Mag. Soc. 15(4), 241 (2005).

[9] W. Y. Jeung, H. K. Kim, and C. B. Park, J. of Kor. Mag. Soc. 16(5), 249 (2006).

[10] H. K Kim, D. W. Chun, J. H. Han, K. B. Kim, and W. Y.
Jeung. Phys. Stat. Sol. (a) 204, 4104 (2007).

[11] Agilent Application Note "Ultralow Impedance Measurement using 2-port Measurement" (2007).

[12] L. Jin, S. S. Yoon et al., J. Magnetics 12, 31 (2007).

[13] Kollu, Pratap, Doung-Young Kim, and Cheol-Gi Kim, J. Magnetics 12, 35 (2007). 